\title{
Just another journal? No, a different one!
}

\author{
Lodewijk Bos • Luis Kun
}

Published online: 5 November 2010

(C) IUPESM and Springer-Verlag (outside the USA) 2010

Why is Health and Technology not just another journal and why does it call itself different? Because the journal will look at both health and technology in a cross-disciplinary and multidirectional way.

In the context of this journal we define Health Technology as

"including drugs, devices, equipment, technical, medical and surgical procedures, the knowledge associated with these in the prevention, diagnosis and treatment of disease as well as in rehabilitation, and the organizational and supportive systems within which care is provided. Included into the definition are the information and communication technologies".

"Health technologies range from single-use devices to the most advanced medical equipment, such as magnetic resonance imaging (MRI) scanners. Technology is used in all types of health facilities, plays a major role in contemporary health care systems and contributes directly to the quality of patient care. It needs to be complemented, however, by good staff training and effective organization of health services where application/use is sought.

Decisions on selecting medical equipment for a health care facility must be supported by evidence and based

L. Bos $(\triangle)$

ICMCC,

Utrecht, The Netherlands

e-mail: lobos@icmcc.org

L. Kun

National Defense University,

Washington, DC, USA on clinical needs, financial resources and the local capacity for effective use. Health technology assessment should be used to support more informed decision-making and contribute to the development of national health care services.

An efficient HTM programme is needed to keep equipment in good working order with maximum effectiveness in terms of clinical use and running costs." [WHO]

There are many journals covering elements included in this definition, however only very few cover the full width. Recent societal developments, due in large parts to changes in the way we communicate, make it necessary to create a journal with the ambition of being cross-disciplinaryopen for all issues and items covered by the above definition - and multidirectional — by any stakeholder for any stakeholder. This approach is not an all-embracing container-for-everything, but a focused and much needed one. Developments in health and technology should be studied, discussed and evaluated from a crossdisciplinary and multidirectional point of view for several reasons:

- Within health sciences specialization is a result of the knowledge explosion and needs to grasp all new phenomena. However, it limits our approach to a patient's condition. A cross-disciplinary approach will increase the diagnostic quality.

- Cooperation in patient's treatment requires an understanding of perspectives from different disciplines. From a patient's point-of-view, there is one patient with one or more complaints, and all available knowledge and experience should be brought together to understand and help the patient. A cross-disciplinary approach will improve cooperation in the chain of health care delivery. 
- Technology that supports or enables cross-disciplinary care has to face the challenges of communication between the different disciplines. On the one hand technology enables new ways of communication, like email or web-chats; on the other, technology does not sufficiently support cross-disciplinary communication, e.g. differences in languages, terminologies and coding schemes between primary and secondary care are not yet solved.

- Technology can no longer be seen as just an instrument, it embeds all kinds of assumptions that reflect the way we live or perceive the world. Norms are being built in, and we have to make explicitly clear which values we like to implement. For example, cross-disciplinary exchange of lab-results must include both norm and method. Since communication is based on trust, the receiver must trust the method and understand the interpretation of the sender. Access to information is nowadays supported and regulated by authorization mechanisms. However, for a responsible security officer it is a challenge to maintain these 'rules': they are too embedded in the systems. Our modern technology has become a social construction. Cross-disciplinary approach is needed to design and validate healthcare technology from different perspectives, like ethical, juridical, social, economic and medical, to understand the assumptions and implications.

- The cross-disciplinary approach is not only necessary within the health area. Technologies developed elsewhere can become a catalyst for change in the way we conduct healthcare as well.

The need for a multidirectional approach comes from a stakeholder analysis. Besides different perspectives, there is the number of people working in healthcare technology all with their proper stake, their proper interest in achieving better care. Government has to deal with conditions for providing good healthcare for a reasonable price for all citizens. Providers and their facilitating partners have to organize healthcare in an economic 'healthy' way. Patients and healthcare professionals have to relate to each other in order to achieve or maintain a status of well-being. Within these three domains (politics, economy and life) each stakeholder has their proper role. The organization of this health and technology eco-system must be studied and discussed explicitly. A multidirectional approach increases the mutual awareness of the broad variety of stakeholders whilst realizing excellent health and technology.

The concept of communication has gone through a major shift in recent history no longer being restricted to humans. Pills, phones, clothes are rapidly becoming monitoring devices, communicating with each other, with systems, with humans and, seemingly, on their own accord.
Ageing and behavior are at the basis of an ever growing number of people who need care and/or cure in any form. This causes a rising demand of resources, human, economic and technical alike, not only in the classically called developed world but also in all other parts of the globe. Increasing economic wellbeing causes both a decline in the number of births and an increase in the number of elderly.

To appropriately deal with these problems we will have to realize, accept and explore our dependency on technology, from process handling to monitoring. Efficiency and error reduction are some of the key arguments used to promote health technologies. In order to achieve those goals, various stakeholders need a basic knowledge about the technology used; at the same time the technology itself has to be safe and efficient.

For all parties involved there are new challenges, following are some examples. Physicians are responsible for the quality of care but will have to accept that providing good healthcare is no longer their sole domain. It depends on versatile, multidisciplinary teams in which clinical engineers play an important role and warrant adequate quality assurance for the health technologies. To select the appropriate tools, physicians need knowledge of software like electronic health records (EHR), software as a service (SaaS), computerized physician order entry (CPOE); engineers are confronted with the development of new sensor technologies (monitoring) and changing hardware requirements (e.g. direct streaming of MRI data), they have to deal with new equipment performance or training demands, or with the upcoming perception of software being a device (EHR); nurses will have to cover a whole broad spectrum of new technologies, from patient records to barcoded medication distribution. All have to face the developments in telemedicine and mobile health (mHealth).

Patients expect doctors to accept lab tests, X-rays, MRI scans which they did not order themselves, which are performed by other providers than their usual ones and delivered electronically. This causes new constraints concerning trust, interoperability and standards, directly linked to the fact that more parts of the delivery of health care (processes, storage or communication) are being digitized.

Technology has become an essential part of our society. And we slowly discover that almost every aspect of technology influences our health and/or wellbeing. The technologies to transport us, cars, trains, planes, are obvious in this sense and much effort is put into making them as safe as possible under strong pressure from the consumer. Different however is for example the situation in the food industry.

The new ways of communication make information about different fields of science and technology more 
easily accessible and also much easier to share which enables certain disciplines to be used in alternative ones. The smartphone was not designed for use in the health and medicine areas but is now rapidly becoming an elementary tool. Broadband cabling is changing from an internet browsing enabler to an essential part of telemedicine and digital homecare. Knowledge of chain management in other areas can be used e.g. in the way we distribute food, but also in the manner we manage hospitals. Of course, these alternative uses will have all kinds of implications, not only within the health field but also economically, legally and ethically. These new and "unexpected" relationships will reshape our view of health technologies.

But it needs an open mind to be able to see these possibilities. It is amazing to see that we spend so much time, money and discussions on the development of an EHR, whereas a fully functional example already exists and has been in use for many years worldwide, monitoring subjects from before conception till after death, taking into account full family history and environmental aspects: the tags in cows' ears, the cattle register.

Patients are facing a different position as well. Due to the internet and the World Wide Web, their access to information has fundamentally changed with direct consequences to their position not only in their own care and cure path but also in prevention matters. New web and mobile applications are changing the concept of selfmanagement and self-help.

The internet has opened the gates of information, allowing patients not only to consume information, but to add to it their own and, even more important, to add and share their own experience: the way persons act with and react to information. This adds new challenges, especially in the field of standards (ontologies, semantics), but also for the delivery and quality of information.

Simultaneously patients more consciously come to realize that the basis of information is data, due to access to their own data (EHR) on the one hand and their "active" participation in the gathering of data (telemedicine, mHealth) on the other.

Developments in information and communication technologies (mobile, internet) are changing the role of the patient and causing the relationship between caregiver and care receiver to go through a major paradigm shift, characterized by terms like patient-centered or participatory medicine. Making both patients and providers accept this paradigm shift will be one of the most important challenges we have to face.

New virtualization technologies, genomics and the field of data mining (made easy by digital storage) will lead to personalized medicine with enormous consequences for patients, their caregivers, the pharma industry and technology providers as well as to new approaches in public health.

The result of the above-mentioned developments is a society that has the ability to empower people, to iron out differences based on century old informational disparities. The new access to information also enables a new way of communication, helping people to realize that only in the rarest occasions their condition/problem is truly and a hundred percent unique, that there always is another person who has been or is in a similar situation, able to help with their experience; and vice versa. Modern technology allows us to seek and hopefully find this person.

These new technologies will be of essential assistance in achieving a new perception in health and medicine, the patient as a partner in their health and wellbeing related processes by offering them tools to assume that position. This will be enhanced by the many other quickly growing fields like genomics, assistive, smart or gaming technology.

This paradigm shift of patients becoming partners will also have consequences in the area of patient safety. First, the patient experience on patient safety must be taken seriously. Instead of statistics, patient safety must be based on patient safety perceptions, in the end as individually as possible. Second, to reduce avoidable errors, data must be exchanged and transformed into relevant information, available for both patient and his healthcare providers. Third, new ways of storing and exchanging information will add a whole new dimension to privacy and security, increasing and implementing privacy enhancement technologies.

The new journal Health and Technology

- will assist navigating these new developments.

- will fill gaps that exist in the education of physicians, nurses (e.g. Biomedical informatics), clinical and biomedical engineers as well as medical physicists and many other health care professions.

- will inform about new technologies that create data (like telemedicine, mHealth) but also about technologies that will help both patient and provider to handle the availability of and the access to data and subsequent information, a growing problem.

- will deal with technology concerning both health care and public health with a strong focus on patient safety, quality and ethical aspects.

- will help understand the consequence of a different way and level of access to information and how it affects relationships of both patient and provider.

- will try to explain which societal and ethical shifts are or will have to be made to optimize the structure and 
organization of our society in concordance with these new developments as well as the economic consequences thereof.

- will play a leading role in easing the ongoing discussion about the influence of (disruptive) technology.

- will pay ample attention to the consequences in policy, workforce, education, training and regulation.

- will give the reader access to newest research, opinions and developments, making them more knowledgeable even outside the areas of their own expertise.

- being the first cross-disciplinary journal it will help to understand how the knowledge society, based on modern technology, influences health in unexpected ways. That the infrastructure of a building can improve health, not only by leaving out asbestos, but by putting in cabling, to name a simple example.

- will look at issues that, on first sight, do not seem related to health.
- last but not least will pay much attention to patientrelated aspects of health technology.

Health and Technology will deal with the technology of health, but also with the health of technology.

Health and Technology will be a peer-reviewed journal according to the highest possible standards. We expect our authors to deliver sound, high quality accounts about their experiences or outstanding reports of first rated research, at the same time aiming at the highest achievable standards of accessibility and understandability for both providers and patients not directly involved in their specific area of expertise.

Lodewijk Bos

Luis Kun

Editors in Chief 